\title{
Lateral-Occipital Sulcus
}

National Cancer Institute

\section{Source}

National Cancer Institute. Lateral-Occipital Sulcus. NCI Thesaurus. Code C32952.

A horizontal groove on the lateral surface of the occipital lobe extending from the accessory lateral occipital sulci to the lunate sulcus that separates the superior occipital gyrus from the inferior occipital gyrus. 